\title{
Capacity Analysis for MIMO Two-Hop Two-Relay Amplify-and-Forward Relaying Systems
}

\author{
Abdulla Firag, Peter J. Smith \\ Department of Electrical and Computer Engineering \\ University of Canterbury, Christchurch, New Zealand
}

\begin{abstract}
This paper presents an ergodic capacity analysis of an amplify-and-forward (AF) multiple-input, multipleoutput (MIMO) two-hop, two relay system. We first derive an expression for the probability density function of an arbitrary eigenvalue of the system. Then, using this result, a closed form expression for the ergodic capacity of the system is derived. We present simulation results to validate our analysis. We also show that the results for a single relay system can be obtained as a special case.
\end{abstract}

\section{INTRODUCTION}

Relaying systems have been of interest since the 1970s [1, 2]. The main objective of such systems is to increase coverage and reduce the need to use high power at the transmitter or source terminal. The basic idea of a relaying system is that the source terminal uses other terminals or relays to forward its information to the destination terminal. In general, there are two types of relaying systems, i.e. non-regenerative and regenerative systems [3], depending on the nature and the complexity of the relays used. In regenerative, also called decode-and-forward (DF), relaying systems, the relay fully decodes the received signal and retransmits the decoded symbol to the destination. Non-regenerative, also called amplify-and-forward (AF), systems use less complex relays that do not perform any sort of decoding. Here the relay simply amplifies the received signal and forwards it to the destination.

Wireless relaying networks have recently been given considerable attention due to their many advantages. Apart from increasing the coverage, relaying networks can also achieve better diversity by using cooperative transmission from several relays $[4,5]$. It is also well known that multiple-input, multiple-output (MIMO) systems can provide better system capacity than singleinput, single-output (SISO) systems [6]. Hence, relaying has recently been extended to MIMO scenarios $[7,8]$ to obtain better system capacity, increases in range, and also to achieve better diversity.

Our main contribution in this paper is to derive an exact expression for the capacity of an AF MIMO twohop two relay system shown in Fig. 1. Further, we will show that these results simplify as a special case to a single relay system. These expressions are useful for gaining further insights into the effect of various system parameters on the performance of the wireless relaying systems.

\section{SYSTEM MODEL}

The source $(S)$, relay $\left(R_{x}\right)$, and destination $(D)$ terminals are equipped with $m, l$ and $n$ antennas respectively. In this model we have assumed that there is no direct link between the source and destination due to the large distance involved. During the first hop, the source terminal transmits (broadcasts) to the relays and in the second hop the relay terminals transmit the amplified signal from the first hop to the destination. We let the normalized channel matrices for the sourceto-relays $\left(S \rightarrow R_{1}, S \rightarrow R_{2}\right)$, and relays-to-destination $\left(R_{1} \rightarrow D, R_{2} \rightarrow D\right)$ links be given by $\boldsymbol{H}_{11}, \boldsymbol{H}_{21} \in \mathcal{C}^{l \times m}$, and $\boldsymbol{H}_{12}, \boldsymbol{H}_{22} \in \mathcal{C}^{n \times l}$, respectively. We assume that the channels are frequency-flat and the entries of the channel matrices are zero mean circularly symmetric complex Gaussian (ZMCSCG) random variables of unit variance. Furthermore, we assume that the relay terminal assists in the communication with the destination terminal using AF mode. In this mode, without decoding or demodulation the relay terminal, $R_{x}$ amplifies the received observation corresponding to the signal from the source by a factor, $N_{x}$ and retransmits it to the destination. In our analysis, $N_{x}$ is chosen so that on average the total transmitted power from $R_{x}$ is $P_{r}$. The received signal at the destination after the 


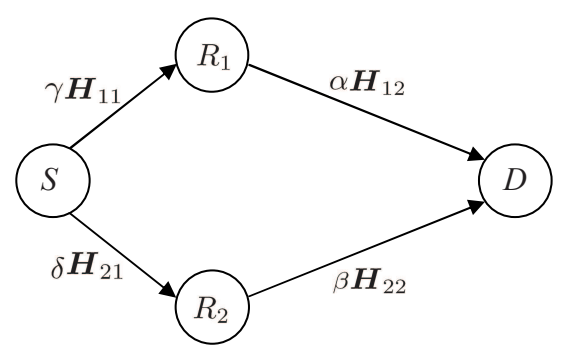

Figure 1: MIMO relay network topology.

two hops is then given by

$$
\begin{aligned}
\boldsymbol{y} & =\overbrace{\left[\begin{array}{ll}
\boldsymbol{H}_{12} & \boldsymbol{H}_{22}
\end{array}\right]}^{\boldsymbol{U}_{1}} \overbrace{\left[\begin{array}{l}
\boldsymbol{H}_{11} \\
\boldsymbol{H}_{21}
\end{array}\right]}^{\boldsymbol{U}_{2}} \boldsymbol{x}+ \\
& {\left[\begin{array}{ll}
\boldsymbol{H}_{12} & \boldsymbol{H}_{22}
\end{array}\right] \underbrace{\left[\begin{array}{cc}
\sigma \frac{\alpha}{N_{1}} \boldsymbol{I}_{l} & \mathbf{0} \\
\mathbf{0} & \sigma \frac{\beta}{N_{2}} \boldsymbol{I}_{l}
\end{array}\right]}_{\boldsymbol{D}} \underbrace{\left[\begin{array}{l}
\boldsymbol{n}_{1} \\
\boldsymbol{n}_{2}
\end{array}\right]}_{\boldsymbol{w}}+\sigma \boldsymbol{n} } \\
& \triangleq \boldsymbol{U}_{1} \boldsymbol{\Gamma} \boldsymbol{U}_{2} \boldsymbol{x}+\boldsymbol{U}_{1} \boldsymbol{D} \boldsymbol{w}+\sigma \boldsymbol{n}
\end{aligned}
$$

where

$$
\boldsymbol{\Gamma}=\left[\begin{array}{cc}
\gamma \sqrt{\frac{P_{t}}{m}} \frac{\alpha}{N_{1}} \boldsymbol{I}_{l} & \mathbf{0} \\
\mathbf{0} & \delta \sqrt{\frac{P_{t}}{m}} \frac{\beta}{N_{2}} \boldsymbol{I}_{l}
\end{array}\right] .
$$

In (1), the parameters $\gamma^{2}, \delta^{2}, \alpha^{2}$ and $\beta^{2}$ are the average powers of the $S \rightarrow R_{1}, S \rightarrow R_{2}, R_{1} \rightarrow D$ and $R_{2} \rightarrow D$ links, respectively, taking into account the different path loss and shadowing effects over the links. The variables $\sigma \boldsymbol{n}_{1}, \sigma \boldsymbol{n}_{2}$ and $\sigma \boldsymbol{n}$ are the noise vectors at $R_{1}, R_{2}$ and $D$, respectively, and $\sqrt{\frac{P_{t}}{m}} \boldsymbol{x}$ is the vector of transmit symbols. The transmit symbols are i.i.d. with $E\left\{\frac{P_{t}}{m} \boldsymbol{x} \boldsymbol{x}^{\dagger}\right\}=\frac{P_{t}}{m} \boldsymbol{I}_{m}$. Hence the total transmit power is $P_{t}$. The noise at relays and destination is modeled as ZMCSCG with $E\left\{\sigma^{2} \boldsymbol{n}_{1} \boldsymbol{n}_{1}^{\dagger}\right\}=\sigma^{2} \boldsymbol{I}_{l}, E\left\{\sigma^{2} \boldsymbol{n}_{2} \boldsymbol{n}_{2}^{\dagger}\right\}=$ $\sigma^{2} \boldsymbol{I}_{l}$, and $E\left\{\sigma^{2} \boldsymbol{n} \boldsymbol{n}^{\dagger}\right\}=\sigma^{2} \boldsymbol{I}_{n}$. With this information, and defining $\boldsymbol{B}=\left(\sigma^{2} \boldsymbol{I}_{n}+\boldsymbol{U}_{1} \boldsymbol{D}^{2} \boldsymbol{U}_{1}^{\dagger}\right)^{1 / 2}$, the received signal at the destination can also be written as

$$
\boldsymbol{y}=\boldsymbol{A x}+\boldsymbol{B} \boldsymbol{v}
$$

where $\boldsymbol{A}=\boldsymbol{U}_{1} \boldsymbol{\Gamma} \boldsymbol{U}_{2}$ and $\boldsymbol{v}$ is a normalized noise vector, which has $\boldsymbol{I}_{n}$ as the covariance matrix.

\section{CAPACITY ANALYSIS}

The ergodic capacity of the system can be calculated by [7] as below, (the factor $1 / 2$ accounts for the fact that information is conveyed to the destination terminal over two time slots [4])

$$
C=\frac{1}{2} E\left\{\log _{2}\left|\boldsymbol{I}_{n}+\boldsymbol{A} \boldsymbol{A}^{\dagger}\left(\boldsymbol{B} \boldsymbol{B}^{\dagger}\right)^{-1}\right|\right\} .
$$

To make the analysis possible, we have to assume that both relays receive the signal with the same signal to noise ratio (SNR), i.e. $\gamma^{2}=\delta^{2}$. This is reasonable when the link distances are similar and is the scenario which tends to maximize the relay capacity. Then using the identity $\operatorname{det}(\boldsymbol{I}+\boldsymbol{A} \boldsymbol{B})=\operatorname{det}(\boldsymbol{I}+\boldsymbol{B} \boldsymbol{A})$, and defining $\widetilde{\boldsymbol{U}}_{1} \triangleq \boldsymbol{U}_{1} \boldsymbol{D}$, the capacity can now be given as

$$
C=\frac{1}{2} E\left\{\log _{2}\left|\boldsymbol{I}_{m}+\rho \boldsymbol{U}_{2}^{\dagger} \widetilde{\boldsymbol{U}}_{1}^{\dagger}\left(\sigma^{2} \boldsymbol{I}_{n}+\widetilde{\boldsymbol{U}}_{1} \widetilde{\boldsymbol{U}}_{1}^{\dagger}\right)^{-1} \widetilde{\boldsymbol{U}}_{1} \boldsymbol{U}_{2}\right|\right\}
$$

where $\rho=\frac{\gamma^{2} P_{t}}{\sigma^{2} m}$. Assume that $n<2 l$, and using the singular value decomposition $\widetilde{\boldsymbol{U}}_{1}=\boldsymbol{E}_{1} \boldsymbol{V} \boldsymbol{E}_{2}^{\dagger}$, the capacity can also be written as

$$
\begin{aligned}
C & =\frac{1}{2} E\left\{\log _{2}\left|\boldsymbol{I}_{m}+\rho \widetilde{\boldsymbol{U}}_{2}^{\dagger} \boldsymbol{V}^{\dagger}\left(\sigma^{2} \boldsymbol{I}_{n}+\boldsymbol{V} \boldsymbol{V}^{\dagger}\right)^{-1} \boldsymbol{V} \widetilde{\boldsymbol{U}}_{2}\right|\right\} \\
& =\frac{n}{2 \ln (2)} \int_{0}^{\infty} \ln (1+\rho \lambda) f(\lambda) d \lambda
\end{aligned}
$$

where $\widetilde{\boldsymbol{U}}_{2}=\boldsymbol{E}_{2}^{\dagger} \boldsymbol{U}_{2}, \lambda$ denotes the non-zero arbitrary eigenvalue of $\widetilde{\boldsymbol{U}}_{2}^{\dagger} \boldsymbol{V}^{\dagger}\left(\sigma^{2} \boldsymbol{I}_{n}+\boldsymbol{V} \boldsymbol{V}^{\dagger}\right)^{-1} \boldsymbol{V} \widetilde{\boldsymbol{U}}_{2}$ and $f(\lambda)$ is the probability density function (pdf) of $\lambda$. Then defining $w_{1}=\sigma^{2} \alpha^{2} / N_{1}^{2}$ and $w_{2}=\sigma^{2} \beta^{2} / N_{2}^{2}$ and using the results in $[9,10]$, the density function, $f(\lambda)$ is given in the following theorem. Note that although the theorem assumes $n \leqslant m<2 l$, all other cases can be handled but are omitted for space reasons.

Theorem: Assume that $n \leqslant m<2 l$, then the density function of the non-zero arbitrary eigenvalue of $\widetilde{\boldsymbol{U}}_{2}^{\dagger} \boldsymbol{V}^{\dagger}\left(\sigma^{2} \boldsymbol{I}_{n}+\boldsymbol{V} \boldsymbol{V}^{\dagger}\right)^{-1} \boldsymbol{V} \widetilde{\boldsymbol{U}}_{2}$ is given by

$$
f(\lambda)=C_{1} \sum_{i=1}^{2 l} \sum_{j=1}^{n}(-1)^{i+j+2 l-n}\left|\boldsymbol{Q}_{i, j+2 l-n}\right| A_{\lambda}(i, j)
$$

where

$$
C_{1}=\frac{1}{n \sigma^{n(n-1)} \Gamma_{n}(m) \Gamma_{n}(n) \Gamma_{l}(l) \Gamma_{l}(l)\left(w_{2}-w_{1}\right)^{l^{2}}}
$$

where $\Gamma_{m}(n)=\prod_{k=1}^{m} \Gamma(n-k+1), \boldsymbol{Q}_{i, j+2 l-n}$ denotes the $(i, j+2 l-n)^{\text {th }}$ minor of $\boldsymbol{Q}$ with elements given in (9) and $A_{\lambda}(i, j)$ is given in (10). The proof of the theorem is in Appendix A. Now the ergodic capacity of the system can be given as

$$
\begin{gathered}
C=\frac{n C_{1}}{2 \ln (2)} \sum_{i=1}^{2 l} \sum_{j=1}^{n}(-1)^{i+j+2 l-n}\left|\boldsymbol{Q}_{i, j+2 l-n}\right| \\
\int_{0}^{\infty} \ln (1+\rho \lambda) A_{\lambda}(i, j) d \lambda .
\end{gathered}
$$


When $\gamma^{2} \neq \delta^{2}$, an approximation, a lower bound and an upper bound for the capacity can be obtained by replacing $\gamma$ and $\delta$ by $\frac{\gamma+\delta}{2}, \min (\gamma, \delta)$ and $\max (\gamma, \delta)$, respectively. These approximations and bounds are quite accurate unless $\gamma^{2} \gg \delta^{2}$ or $\gamma^{2} \ll \delta^{2}$. However, in these cases it is usually preferable to use a single relay system. Hence the analysis is useful for most cases of interest.

Using the result given in (8), the ergodic capacity for a single MIMO relaying system for $n \leqslant m<2 l$ can be obtained by setting appropriate parameters. Details are given in Appendix B.

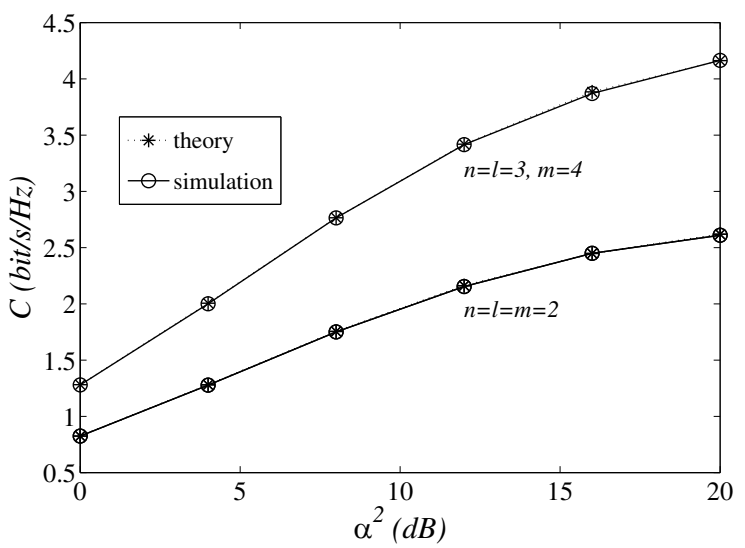

Figure 2: Mean capacity $\left(\gamma^{2}=\delta^{2}=10 \mathrm{~dB}, \alpha^{2}=\beta^{2}-4 \mathrm{~dB}\right.$ and $\left.P_{t}=P_{r}=\sigma^{2}=1\right)$.

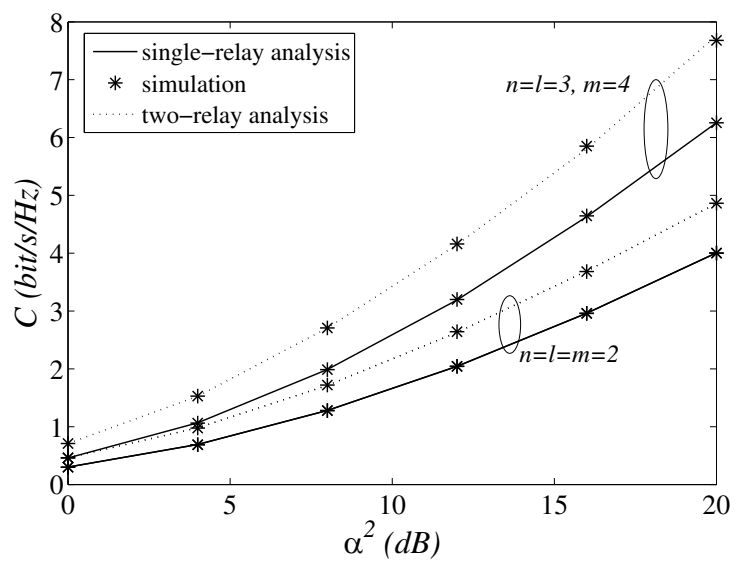

Figure 3: Relay system capacity with one and two relays $\left(\gamma^{2}=\delta^{2}=\alpha^{2} \approx \beta^{2}\right.$ and $\left.P_{t}=P_{r}=\sigma^{2}=1\right)$.

\section{RESULTS}

The results produced in this paper are validated by using Monte Carlo simulation. In all the results given, we let $P_{t}=P_{r}=\sigma^{2}=1$ and thus the relays' amplification factors are given by, $N_{1}=\sqrt{l\left(\gamma^{2}+1\right)}$ and $N_{2}=\sqrt{l\left(\delta^{2}+1\right)}$. The value of $N_{x}$ is chosen so that on average the total transmitted power from a relay is $1\left(P_{r}=1\right)$. First the ergodic capacity of the system shown in Fig. 1 is given for different system variables in Fig. 2. The analytical results show a perfect agreement with the simulation results.

We also show that the capacity expression derived in this paper can be used for a single relay system by the simple expedient of setting $\beta=0$. Derivations and results for this case are given in Appendix B. The results are shown in Fig. 3 for both two relay and single relay systems. Excellent agreement is observed between the simulations and analysis of the single relay system. The results also show that the system with two relays provides better capacity compared to a single relay system, due to extra diversity.

The results derived in the paper assume $\gamma^{2}=\delta^{2}$. However, when $\gamma^{2} \neq \delta^{2}$, an approximation, a lower bound and an upper bound for the capacity can be obtained as mentioned before. Figure 4 shows the capacity and bounds for the relaying system with two relays when $\gamma^{2} \neq \delta^{2}$. The results show that the approximation, lower bound and upper bound for the capacity are in good agreement with the simulated results.

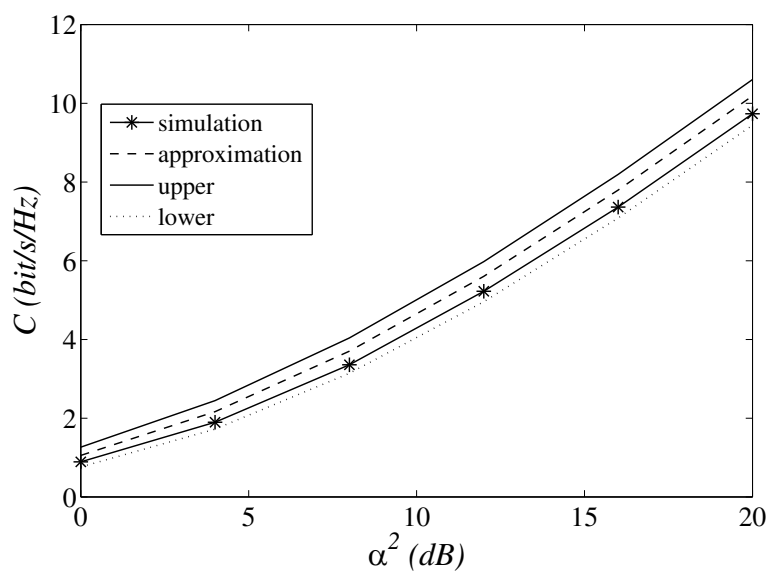

Figure 4: Capacity bounds for a relaying system with two relays $\left(\gamma^{2}=\alpha^{2}, \beta^{2}=\alpha^{2}-5 \mathrm{~dB}, \delta^{2}=\gamma^{2}+5 \mathrm{~dB}, P_{t}=\right.$ $P_{r}=\sigma^{2}=1$ and $\left.n=l=4, m=5\right)$.

\section{CONCLUSIONS}

In this paper we have derived an exact expression for the capacity of the AF MIMO two-hop two relay system shown in Fig. 1. We first derived an expression for the probability density function of the arbitrary eigenvalue of the system. Then, using this result, 


$$
\boldsymbol{Q}_{i, j}= \begin{cases}(j-l+i)_{l-i} w_{1}^{j-l+i-1} & i=1, \ldots, l, j=1, \ldots, 2 l-n \\ (j-2 l+i)_{2 l-i} w_{2}^{j-2 l+i-1} & i=l+1, \ldots, 2 l, j=1, \ldots, 2 l-n \\ \sum_{k=0}^{l-i} \frac{(l-i) !}{k !(l-i-k) !}(l-n+i)_{l-i-k} w_{1}^{l-n+i-k-1} & \\ \Gamma(m+j-2 l) I_{A}\left(j, k, w_{1}\right) & i=1, \ldots, l, j=2 l-n+1, \ldots, 2 l \\ \sum_{k=0}^{2 l-i} \frac{(2 l-i) !}{k !(2 l-i-k) !}(-n+i)_{2 l-i-k} w_{2}^{-n+i-k-1} & \\ \Gamma(m+j-2 l) I_{A}\left(j, k, w_{2}\right) & i=l+1, \ldots, 2 l, j=2 l-n+1, \ldots, 2 l\end{cases}
$$

where $I_{A}(j, k, w)=\sum_{p=0}^{2 l-j} \frac{(2 l-j) !}{p !(2 l-j-p) !} \sigma^{2(2 l-j-p)} w^{n+j+k+p-2 l} \Gamma(n+j+k+p-2 l)$ and $(x)_{y}$ is the Pochhammer symbol.

$$
A_{\lambda}(i, j)= \begin{cases}\sum_{k=0}^{l-i} \frac{(l-i) !}{k !(l-i-k) !}(l-n+i)_{l-i-k} w_{1}^{l-n+i-k-1} \lambda^{m+j-n-1} & i=1, \ldots, l \\ I_{B}\left(k, w_{1}\right) & i=l+1, \ldots, 2 l \\ \sum_{k=0}^{2 l-i} \frac{(2 l-i) !}{k !(2 l-i-k) !}(-n+i)_{2 l-i-k} w_{2}^{-n+i-k-1} \lambda^{m+j-n-1} & \\ I_{B}\left(k, w_{2}\right) & i=,\end{cases}
$$

where $I_{B}(k, w)=\sum_{p=0}^{m} \frac{m !}{p !(m-p) !} \sigma^{2(m-p)} e^{-\lambda} 2\left(\lambda \sigma^{2} w\right)^{(n+p-m+k) / 2} K_{n+p-m+k}\left(2 \sqrt{\lambda \sigma^{2} / w}\right)$ and $K_{x}$ is the modified Bessel function of the second kind.

a closed form expression for the ergodic capacity of the system is derived. The analysis is verified using Monte Carlo simulation and shows perfect agreement with the simulation. We also showed that these results can be used to obtain the capacity for a single relay system by setting appropriate parameters.

\section{APPENDIX}

\section{A. Proof of the Theorem}

For the case $n \leqslant m<2 l$, the matrix $\widetilde{\boldsymbol{U}}_{1}^{\dagger} \widetilde{\boldsymbol{U}}_{1}$ is pseudo-Wishart [11]. Now, let $\widetilde{\boldsymbol{U}}_{1}^{\dagger} \widetilde{\boldsymbol{U}}_{1}$ have ordered nonzero eigenvalues, $\nu_{1}>\ldots>\nu_{n}>0$. In this case the diagonal elements of the matrix, $\boldsymbol{D}^{2}$ in (1), are not all distinct. Hence using the results in $[9,12]$ and the following identities on multiple derivatives,

1. If $y=x^{n}$, then the $k^{\text {th }}$ derivative of $y, y^{(k)}=$ $(n-k+1)_{k} x^{n-k}$, and

2. If $y=x^{n} e^{-s / x}$, then $k^{\text {th }}$ derivative of $y, y^{(k)}=$ $e^{-s / x} \sum_{i=0}^{k} \frac{k !}{i !(k-i) !}(n-k+1)_{k-i} s^{i} x^{n-k-i}$,

the density of the unordered nonzero eigenvalues of $\widetilde{\boldsymbol{U}}_{1}^{\dagger} \widetilde{\boldsymbol{U}}_{1}$ can be evaluated as

$$
f(\boldsymbol{\nu})=\frac{\prod_{k<p}^{n}\left(\nu_{p}-\nu_{k}\right)|\boldsymbol{K}|}{n ! \Gamma_{n}(n) \Gamma_{l}(l) \Gamma_{l}(l)\left(w_{2}-w_{1}\right)^{l^{2}}} .
$$

In (11), $\boldsymbol{K}$ is a $2 l \times 2 l$ matrix with entries given in (12). Now, let $\boldsymbol{P}=\boldsymbol{V}^{\dagger}\left(\sigma^{2} \boldsymbol{I}_{n}+\boldsymbol{V} \boldsymbol{V}^{\dagger}\right)^{-1} \boldsymbol{V}$, where $\boldsymbol{V}$ is defined in (5), and for $n \leqslant m<2 l, \boldsymbol{P}$ can be given as

$$
\boldsymbol{P}=\operatorname{diag}\{\mu_{1}, \ldots, \mu_{n}, \underbrace{0, \ldots, 0}_{2 l-n}\},
$$

where $\mu_{k}=\frac{\nu_{k}}{\nu_{k}+\sigma^{2}}$. Then, the capacity can also be expressed as

$$
C=\frac{1}{2} E\left\{\log _{2}\left|\boldsymbol{I}_{m}+\rho \widehat{\boldsymbol{U}}_{2}^{\dagger} \widehat{\boldsymbol{P}} \widehat{\boldsymbol{U}}_{2}\right|\right\}
$$

where $\widehat{\boldsymbol{U}}_{2} \in \mathcal{C}^{n \times m}$ has i.i.d ZMCSCG entries with unit variance and $\widehat{\boldsymbol{P}}=\operatorname{diag}\left\{\mu_{1}, \ldots, \mu_{n}\right\}$. Note that $\widehat{\boldsymbol{P}}$ and $\widehat{\boldsymbol{U}}_{2}$ are simply re-sized versions of $\boldsymbol{P}$ and $\widetilde{\boldsymbol{U}}_{2}$. The matrix $\widehat{\boldsymbol{P}}^{1 / 2} \widehat{\boldsymbol{U}}_{2} \widehat{\boldsymbol{U}}_{2}^{\dagger} \widehat{\boldsymbol{P}}^{1 / 2}$ is central complex Wishart [13]. Then using the result in [9] for the pdf of the non-zero arbitrary eigenvalue for the Wishart case, the conditional pdf of a non-zero arbitrary eigenvalue of $\widehat{\boldsymbol{P}}^{1 / 2} \widehat{\boldsymbol{U}}_{2} \widehat{\boldsymbol{U}}_{2}^{\dagger} \widehat{\boldsymbol{P}}^{1 / 2}$ can be obtained as

$$
f(\lambda \mid \boldsymbol{\mu})=\frac{\sum_{r=1}^{n}\left|\boldsymbol{G}^{1}\right|}{n \Gamma_{n}(m) \prod_{k=1}^{n} \mu_{k}^{m-n+1} \prod_{k<p}^{n}\left(\mu_{p}-\mu_{k}\right)} .
$$

In (16), $G^{1}$ is a $n \times n$ matrix with entries

$$
\boldsymbol{G}_{i, j}^{1}=\left\{\begin{array}{ll}
\lambda^{m-n+i-1} e^{-\lambda / \mu_{j}} & i=r, j=1, \ldots, n \\
\frac{\Gamma(m-n+i)}{\mu_{j}^{n-m-i}} & i \neq r, j=1, \ldots, n
\end{array} .\right.
$$

Then the conditional pdf $f(\lambda \mid \boldsymbol{\nu})$ can be obtained by substituting $\mu_{k}=\left(\frac{\nu_{k}}{\sigma^{2}+\nu_{k}}\right)$ in (16). Now the joint pdf 


$$
\boldsymbol{K}_{i, j}= \begin{cases}(j-l+i)_{l-i} w_{1}^{j-l+i-1} & i=1, \ldots, l, j=1, \ldots, 2 l-n \\ e^{-\nu_{j-2 l+n} / w_{1}} & \\ \sum_{k=0}^{l-i} \frac{(l-i) !}{k !(l-i-k) !}(l-n+i)_{l-i-k} \nu_{j-2 l+n}^{k} w_{1}^{l-n+i-k-1} & i=1, \ldots, l, j=2 l-n+1, \ldots, 2 l \\ (j-2 l+i)_{2 l-i} w_{2}^{j-2 l+i-1} & i=l+1, \ldots, 2 l, j=1, \ldots, 2 l-n \\ e^{-\nu_{j-2 l+n} / w_{2}} & \\ \sum_{k=0}^{2 l-i} \frac{(2 l-i) !}{k !(2 l-i-k) !}(-n+i)_{2 l-i-k} \nu_{j-2 l+n}^{k} w_{2}^{-n+i-k-1} & i=l+1, \ldots, 2 l, j=2 l-n+1, \ldots, 2 l\end{cases}
$$

$$
\mathbf{\Psi}_{i, j}= \begin{cases}(j-l+i)_{l-i} w_{1}^{j-l+i-1} & i=1, \ldots, l, j=1, \ldots, 2 l-n \\ (j-2 l+i)_{2 l-i} w_{2}^{j-2 l+i-1} & i=l+1, \ldots, 2 l, j=1, \ldots, 2 l-n \\ \sum_{k=0}^{l-i} \frac{(l-i) !}{k !(l-i-k) !}(l-n+i)_{l-i-k} w_{1}^{l-n+i-k-1} \Gamma(m+j-2 l) & i=1, \ldots, l, j=2 l-n+1, \ldots, 2 l \text { and } \\ I_{A}\left(j, k, w_{1}\right) & j \neq r+2 l-n \\ \sum_{k=0}^{2 l-i} \frac{(2 l-i) !}{k !(2 l-i-k) !}(-n+i)_{2 l-i-k} w_{2}^{-n+i-k-1} \Gamma(m+j-2 l) & i=l+1, \ldots, 2 l, j=2 l-n+1, \ldots, 2 l \text { and } \\ I_{A}\left(j, k, w_{2}\right) & j \neq r+2 l-n \\ \sum_{k=0}^{l-i} \frac{(l-i) !}{k !(l-i-k) !}(l-n+i)_{l-i-k} w_{1}^{l-n+i-k-1} \lambda^{m+j-2 l-1} & i=1, \ldots, l, j=2 l-n+1, \ldots, 2 l \text { and } \\ I_{B}\left(k, w_{1}\right) & j=r+2 l-n \\ \sum_{k=0}^{2 l-i} \frac{(2 l-i) !}{k !(2 l-i-k) !}(-n+i)_{2 l-i-k} w_{2}^{-n+i-k-1} \lambda^{m+j-2 l-1} & i=l+1, \ldots, 2 l, j=2 l-n+1, \ldots, 2 l \text { and } \\ I_{B}\left(k, w_{2}\right) & j=r+2 l-n\end{cases}
$$

$f(\lambda, \boldsymbol{\nu})=f(\boldsymbol{\nu}) f(\lambda \mid \boldsymbol{\nu})$ can be obtained as

$$
\begin{aligned}
f(\lambda, \boldsymbol{\nu})= & \frac{1}{n n ! \sigma^{n(n-1)} \Gamma_{n}(m) \Gamma_{n}(n) \Gamma_{l}(l) \Gamma_{l}(l)\left(w_{2}-w_{1}\right)^{l^{2}}} \\
& \sum_{r=1}^{n} \prod_{k=1}^{n} \frac{\left(\sigma^{2}+\nu_{k}\right)^{m}}{\nu_{k}^{m-n+1}}|\boldsymbol{K}|\left|\boldsymbol{G}^{1}\right| \\
\triangleq & C_{0} \sum_{r=1}^{n} \prod_{k=1}^{n} \xi\left(\nu_{k}\right)|\boldsymbol{K}|\left|\boldsymbol{G}^{1}\right|
\end{aligned}
$$

Now $f(\lambda)$ can be obtained by integrating over all $\nu_{k}$ by using the method described in Lemma 2 of [10] as,

$$
\begin{aligned}
f(\lambda) & =C \sum_{r=1}^{n} \int_{0}^{\infty} \ldots \int_{0}^{\infty} \prod_{k=1}^{n} \xi\left(\nu_{k}\right)|\boldsymbol{K}|\left|\boldsymbol{G}^{1}\right| d \nu_{1} \ldots d \nu_{n} \\
& =\underbrace{C n !}_{C_{1}} \sum_{r=1}^{n}|\boldsymbol{\Psi}|
\end{aligned}
$$

where $\boldsymbol{\Psi}$ is a $2 l \times 2 l$ matrix with entries given in (13). Finally, we obtain the result in the theorem by using the Laplace expansion of (19).

\section{B. Single Relaying System}

The ergodic capacity for a single MIMO relaying system for $n \leqslant m<2 l$ can be obtained from the results given in (8). By letting $\beta^{2}=0$ (removing the link between source and destination through $R_{2}$ ) in (8) the capacity for single relaying system can be given as

$$
\begin{gathered}
C=\frac{n C_{1}}{2 \ln (2)} \sum_{i=1}^{2 l} \sum_{j=1}^{n}(-1)^{i+j+2 l-n}\left|\boldsymbol{Q}_{i, j+2 l-n}\right| \\
\int_{0}^{\infty} \ln (1+\rho \lambda) A_{\lambda}(i, j) d \lambda .
\end{gathered}
$$

In (20), $\boldsymbol{Q}$ and $A_{\lambda}(i, j)$ have much simpler results than $(9),(10)$ and they are given in (21) and (22), respectively.

\section{References}

[1] E. C. V. D. Meulen, "Three-terminal communication channels," Adv. Appl. Prob., vol. 3, no. 1, pp. 120-154, 1971.

[2] T. Cover and A. Gamal, "Capacity theorems for the relay channel," IEEE Trans. Inf. Theory, vol. 25, no. 5, pp. 572-584, Sep 1979.

[3] J. Boyer, D. Falconer, and H. Yanikomeroglu, "A theoretical characterization of the multihop wireless communications channel with diversity," in Proc. IEEE Global Telecommunications Conf., vol. 2, 2001, pp. 841-845. 


$$
\boldsymbol{Q}_{i, j}=\left\{\begin{array}{ll}
(j-l+i)_{l-i} w_{1}^{j-l+i-1} & i=1, \ldots, l, j=1, \ldots, 2 l-n \\
0 & i=l+1, \ldots, 2 l, j=1, \ldots, 2 l-n, i \neq 2 l+1-j \\
(j-2 l+i)_{2 l-i} & i=l+1, \ldots, 2 l, j=1, \ldots, 2 l-n, i=2 l+1-j \\
\sum_{k=0}^{l-i} \frac{(l-i) !}{k !(l-i-k) !}(l-n+i)_{l-i-k} w_{1}^{l-n+i-k-1} & \\
\Gamma(m+j-2 l) I_{A}\left(j, k, w_{1}\right) & i=1, \ldots, l, j=2 l-n+1, \ldots, 2 l \\
0 & i=l+1, \ldots, 2 l, j=2 l-n+1, \ldots, 2 l
\end{array},\right.
$$

where $I_{A}(j, k, w)$ is given in (9).

$$
A_{\lambda}(i, j)=\left\{\begin{array}{ll}
\sum_{k=0}^{l-i} \frac{(l-i) !}{k !(l-i-k) !}(l-n+i)_{l-i-k} w_{1}^{l-n+i-k-1} \lambda^{m+j-n-1} & \\
I_{B}\left(k, w_{1}\right) & i=1, \ldots, l \\
0 & i=l+1, \ldots, 2 l
\end{array},\right.
$$

where $I_{B}(k, w)$ is given in (10).

[4] R. U. Nabar, H. Bolcskei, and F. Kneubuhler, "Fading relay channels: Performance limits and space-time signal design," IEEE J. Select. Areas Commun., vol. 22, pp. 1099-1109, Aug. 2004.

[5] A. Sendonaris, E. Erkip, and B. Aazhang, "User cooperation diversity - part 1: System description," IEEE Trans. Commun., vol. 51, pp. 19271938, Nov. 2003.

[6] G. J. Foschini and M. J. Gans, "On limits of wireless communication in a fading environment when using multiple antennas," Wireless Personal Commun., vol. 6, no. 3, pp. 311-335, Mar. 1998.

[7] M. Herdin, "MIMO amplify-and-forward relaying in correlated MIMO channels," in Proc. Int. Conf. on Inform. Commun. and Signal Processing, Bangkok, Thailand, Dec. 6-9, 2005, pp. 796-800.

[8] X. Tang and Y. Hua, "Optimal design of nonregenerative MIMO wireless relays," IEEE Transactions on Wireless Communications, vol. 6, no. 4, pp. 1398-1407, 2007.

[9] A. Maaref and S. Aïssa, "Eigenvalue distributions of Wishart-type random matrices with application to the performance analysis of MIMO MRC systems," IEEE Transactions on Wireless Communications, vol. 6, no. 7, pp. 2678-2689, 2007.

[10] H. Shin, M. Z. Win, J. H. Lee, and M. Chiani, "On the capacity of doubly correlated MIMO channels," IEEE Trans. Wireless Commun., vol. 5, pp. 2253-2265, Aug. 2006.

[11] J. A. Díaz-García, R. G. Jáimez, and K. V. Mardia, "Wishart and pseudo-wishart distributions and some applications to shape theory," J. Multivar. Anal., vol. 63, no. 1, pp. 73-87, 1997.

[12] M. Chiani, M. Z. Win, and H. Shin, "Capacity of MIMO systems in the presence of interference," in Proc. IEEE Global Telecommunications Conf., San Francisco, California, USA, Nov. 30-Dec. 1, 2006 .

[13] R. K. Mallik, "The pseudo-Wishart distribution and its application to MIMO systems," IEEE Trans. Inf. Theory, vol. 49, pp. 2761-2769, 2003. 